\title{
Melanocortin-4 receptor mutations are a frequent and heterogeneous cause of morbid obesity
}

\author{
Christian Vaisse, ${ }^{1,2,3}$ Karine Clement,, ${ }^{1,2}$ Emmanuelle Durand, ${ }^{2}$ \\ Serge Hercberg, ${ }^{4}$ Bernard Guy-Grand, ${ }^{2}$ and Philippe Froguel ${ }^{1,2}$ \\ ${ }^{1}$ Centre National de la Recherche Scientifique, UPRES A 8090, Institute of Biology of Lille, Lille, France \\ ${ }^{2}$ Laboratoire de Nutrition et Service de Médecine et Nutrition, Hôtel-Dieu, Paris, France \\ ${ }^{3}$ Department of Medicine and Diabetes Research Center, University of California San Francisco, San Francisco, \\ California, USA \\ ${ }^{4}$ Conservatoire National des Artes et Metiers, Paris, France
}

Address correspondence to: Christian Vaisse, University of California San Francisco,

Department of Medicine and Diabetes Research Center, Room HSW1113, 513 Parnassus Avenue,

San Francisco, California 94143-0573, USA. Phone: (415) 514-0530; Fax: (415) 476-1660; E-mail: vaisse@medicine.ucsf.edu.

Received for publication December 22, 1999, and accepted in revised form June 7, 2000.

By integrating an agonist satiety signal, provided by alpha-melanocyte-stimulating hormone ( $\alpha$ $\mathrm{MSH}$ ), and an antagonist signal, provided by agouti-related protein (AGRP), the melanocortin-4 receptor (MC4-R) is a key element in the hypothalamic control of food intake. Inactivation of the gene encoding this $\mathrm{G}$ protein-coupled receptor causes obesity in mice. In humans, frameshift mutations in $M C 4-R$ cause an early-onset dominant form of obesity in two families. In this study we find a high frequency (4\%) of rare heterozygous $M C 4-R$ mutations in a large population of morbidly obese patients. No such mutations were found in controls. By analyzing the phenotypes of the probands carrying these mutations, we demonstrate that these patients display a common, nonsyndromic form of obesity. Interestingly, functional analysis of the mutant receptors indicates that obesity-associated defects in $M C 4-R$ range from loss of function to constitutive activation. Transmission of these mutations in the families of the carriers indicates a variable expressivity that is not related to the functional severity of the mutations. This variable expressivity of MC4-R-associated obesity is not due to variations in genes for $\alpha-M S H$ or AGRP. Taken together, these results demonstrate that MC4-R mutations are a frequent but heterogeneous genetic cause of morbid obesity.

J. Clin. Invest. 106:253-262 (2000).

\section{Introduction}

Obesity is the most common and rapidly growing nutritional problem in Westernized countries. By dramatically raising mortality (1) and the risk of morbidity from hypertension, dyslipidemia, diabetes mellitus, and cardiovascular diseases (2), this condition has become one of the major public health concerns for the 21st century $(3,4)$. Obesity is a complex multifactorial disease caused by the interaction of genetic and environmental factors $(5,6)$. The potential implication of genetic factors in the development of human obesity is well demonstrated by the description of five monogenic forms of human obesity (7-15). The genes implicated in these forms of obesity encode proteins of the leptin axis and brain-expressed targets of leptin involved in the melanocortin pathway. They include leptin $(7,8)$, the leptin receptor (9), proconvertase 1 (11), pro-opiomelanocortin (POMC) (10), and the melanocortin-4 receptor (MC4-R) $(12-14,15)$. Except for MC4-R, mutations in these genes cause rare, recessive, syndromic forms of obesity, associated with multiple endocrine abnormalities $(7,10,11,13-15)$.

MC4-R is a 332-amino acid protein encoded by a single exon gene localized on chromosome 18q22 (16, 17). MC4-R belongs to the family of seven transmembrane G protein-coupled receptors (GPCR) and sig- nals through activation of adenylate cyclase (16). MC4-R integrates an agonist signal provided by alpha-melanocyte-stimulating hormone $(\alpha-\mathrm{MSH})$ (18), a melanocortin produced by proconvertase 1-dependent cleavage of POMC (19) and an antagonist signal provided by agouti-related protein (AGRP) expressed in the hypothalamic arcuate nucleus, in neurons distinct from the POMC neurons $(20,21)$. The importance of MC4-R in body-weight regulation is clearly demonstrated by the obese phenotype of the homozygous $M C 4-R$-deficient mice (22). Heterozygous $M C 4-R^{+/-}$mice present an intermediate average increase in weight, females being more severely affected than males (22).

We have reported a case of human obesity associated with a frameshift mutation in MC4-R (12). This case differed from the previously described monogenic forms of obesity by a dominant transmission of the morbidly obese phenotype and by the absence of associated endocrine symptoms (12). The description of a similar case (13) and the report of several MC4- $R$ missense and nonsense mutations in $1 \%$ of two cohorts of extremely obese children (14) and adults (15) suggest that such mutations could be a more frequent cause of obesity than the syndromic forms due to the other 
genes. Limited information on the function of most of the missense mutations as well as incomplete phenotypic characterization of the patients and their families have not yet allowed a case to be built for the overall importance of MC4-R mutations in human obesity.

\section{Methods}

Subjects and phenotypes. We randomly selected 209 subjects from a cohort of morbidly obese unrelated probands of independent families. DNA and clinical and metabolic data from these probands and their families were collected as described (23). Briefly, they were recruited through the Department of Nutrition at the Hôtel-Dieu Hospital in Paris or through a toll-free number in France. In their weight history, all these subjects had reached the diagnostic cut-off point for morbid obesity, i.e., body mass index (BMI) $>40 \mathrm{~kg} / \mathrm{m}^{2}$. Obesity was assessed by BMI, Z-scores (standard deviation over mean BMI at a given age and sex for a French reference population), retrospective weight history, and leptin levels. Metabolic complications of obesity were evaluated by carbohydrate, insulin, and lipid measurements. For some of the subjects we measured body composition by biphotonic absorptiometry (DXA; Hologic Inc., Waltham, Massachusetts, USA) and resting metabolic rate by indirect calorimetry (Deltatrac II MBM200; Datex-Ohmeda, Trappes, France). Blood sampling was also performed after an overnight fast for hormone measurements. The clinical data of the 209 morbidly obese subjects were as follows: sex $\mathrm{M} / \mathrm{F}=$ $50: 159$, age $42 \pm 12.0$ range [12-69], $\mathrm{BMI}=51 \pm 7.5$ [32-74], maximal BMI reached $=54 \pm 8.5 \mathrm{~kg} / \mathrm{m}^{2}$. Sixty (32\%) were obese during infancy and 123 [64\%] were obese at puberty.

In a subgroup of 91 subjects, quantitative food intake was assessed by dietary history performed by specifically trained dieticians. A qualitative food-intake evaluation was performed for 187 probands by the Three-Factor Eating Questionnaire (TFEQ), a psychometric instrument developed for the study of eating behavior (24). This autoquestionnaire of 51 items measures three dimensions of human eating behavior: restraint, disinhibition, and hunger. Restrained eating (Factor I, 21 items) is defined as the tendency to restrict food intake in order to control body weight. Disinhibition (Factor II, 16 items) is the inability to resist emotional and social eating cues. Hunger, factor III (14 items) is the subjective feeling of hunger. The subjects completed the dietary questionnaire before undergoing health examination and dietary inquiry. Data concerning estimation of their physical activity were also obtained in 184 of 209 subjects using the Baecke questionnaire (25).

Two control groups of nonobese subjects were studied. In the first control group ( $\operatorname{control} 1, n=254$ ), individuals were randomly chosen among 3,000 individuals participating in a French study on the efficacy of daily supplementation with antioxidant vitamins and minerals in reducing the major health problems and the causes of premature death in a large population of healthy volunteers (SU-VI-MAX study) (26). The second group of 112 nonobese, nondiabetic controls (control 2) were spouses of patients from a well-established, previously described cohort of French type 2 diabetic families (23).

In both control groups, none of the subjects had reached the diagnostic cut-off point for obesity (BMI > 30) (see Table 2 for subject characteristics). In control group 2, 7.5\% of subjects were overweight (BMI, 27-30). Informed consent was obtained from all subjects and the protocols were approved by the Ethics Committee of Paris (Hôtel-Dieu and Cochin). Genomic DNA was extracted from peripheral leukocytes for all subjects.

PCR-single-stranded conformational polymorphism analysis. The coding portion of the MC4-R gene was amplified using five primer pairs. The $\alpha$-MSH-encoding sequence of the POMC gene and each exon of hAGRP were amplified with one primer pair. PCR reactions were performed in a $25-\mu \mathrm{L}$ volume containing $1 \times$ GeneAmp PCR Buffer II using AmpliTaq DNA Polymerase in the presence of $\left[{ }^{32} \mathrm{P}\right]$-dCTP. Primer pairs forward $\left(5^{\prime} \rightarrow 3^{\prime}\right)$ and reverse $\left(5^{\prime} \rightarrow 3^{\prime}\right)$, length of the PCR products, annealing temperatures, and $\mathrm{MgCl}^{2}$ concentration in the PCR reaction are indicated as follows: MCAF (ATCAATTCAGGGGGACACTG)/MCAR (CATGGGTGAATGCAGATTCT), MCBF (GTGATTGTGGCAATAGCCAA)/MCBR (TCCACTGCAATTGAAAGCAG), MCCF (TGTAGCTCCTTGCTTGCATC)/MCCR (GGCCATCAGGAACATGTGGA), MCDF (ACCATGTTCTTCACCATGCTG)/MCDR (GAGACATGAAGCACACACAA), MCEF (CCATTCTTCCTCCACTTAAT)/MCER (TGCATGTTCCTATATTGCGTG), $55^{\circ} \mathrm{C}, 1.5 \mathrm{mM}$; POMCF (GAAGACTGCGGCCCGCTGCCT)/POMCR (CGTCATCGGCAGGGCCGTCG), $65^{\circ} \mathrm{C}, \quad 1.5 \mathrm{mM}$ (10\% DMSO); ARTAF (TCTTCCTGCTGAGCCAG)/ARTAR (GTCCCACCCTTGCTCACACT), $65^{\circ} \mathrm{C}, 0.5 \mathrm{mM}$ (2.5\% formamide); ARTBF (CCTGCCCTCACATCCTCTG)/ARTBR (CCTTACCCTTTTCCCTGAGC), $65^{\circ} \mathrm{C}, 0.5 \mathrm{mM}$; ARTCF (GTGCTGGTTCCCCACCCCTG)/ARTCR (CCTACCCTAGCCCCGAC$\mathrm{CCT}), 65^{\circ} \mathrm{C}, 1 \mathrm{mM}$ (2.5\% formamide). The radiolabeled PCR products were diluted 1:5 in formamide buffer, and electrophoresed on glycerol-free and 10\% (vol/vol) glycerol-containing nondenaturing $5 \%$ polyacrylamide gels at room temperature or $4^{\circ} \mathrm{C}$, respectively. The gels were electrophoresed at 6-20 W for 4-7 hours, dried, and autoradiographed overnight.

Cloning and sequencing. Sequence of the variant was first determined by reamplification of the PCR product and direct sequencing on an ABI 377. The variant and wild-type genes were amplified from genomic DNA using primers MC4AF and MC4ER and cloned into the pcDNA3/MC4-R/V5/His/Topo expression vector (Invitrogen, Carlsbad, California, USA).

PCR-RFLP analysis. For each variant found to result in a frameshift or missense mutation, a PCR-RFLP assay was designed. Single-stranded conformational polymorphism (SSCP) primers were used for amplification when the mutation was found to introduce or delete a restriction site for a commercially available restriction 
enzyme. For the other mutations, primers with a sequence mismatch in the $3^{\prime}$ region were designed in order to create artificial mutation conditional restriction sites. The primer used, as well as the PCR conditions, are indicated next, followed by the restriction enzyme used, with mismatched bases underlined: mutation Thr11Ser, MCAF/MCAR, cut CaC8I (only mutant cuts); mutation Arg18Cys, MCAF/MCAR, cut AciI (only wild-type [WT]) cuts); mutation 47-48insG, (GCACACTTCTCTGCACCGCTG)/MCAR, cut MwoI (only WT cuts); mutation Val103Ile, MCBF/MC4RBB(TCTGTACTGTTTAATAGGGTGTTG), cut HinCII (only WT [most frequent] cuts); mutation Thr150Ile, MC4RC0 (TTGCAGTGGACAGGTACGTTA)/MCCR, cut MaeIII (only WT cuts); mutation Ile170Val, MCCF/MC4RCx (GCAAGCTGCCCAGATACAAGTTA), cut MaeIII (only mutant cuts); mutation Arg165Trp, MCCF/MCCR, cut AciI (only WT cuts); mutation Ile251Leu, MC4RD0 (GCGATTACCTTGACCAGCCTG)/MCDR cut CaC8I (only mutant cuts); mutation Leu250Gln, MCDF/MC4RDy (GCAGACAACAAAGACGCCACETC), cut DdeI (only WT cuts); mutation Ile301Thr, MCEF/MC4RE0 (GTTCTTGACTCCGGAGTGCÉTAA), cut DdeI (only mutant cuts).

MC4-R activity and binding assay in stably transfected cells. Stable cell lines were established by transfecting HEK 293 cells using Effectene (QIAGEN, Hilden, Germany) and selected in $1 \mathrm{mg} / \mathrm{mL}$ Geneticin (Life Technologies, Merelbecke, Germany) with the cloned sequence confirmed and wild-type and mutant MC4-R. To avoid clonal variations, pools of stable transformants were used in the assay. Equivalent expression of receptor by the different pools of stably transformed cells was assessed by Northern blot. The melanocortin receptor activation assay was adapted from Lu et al. (27) using pCRE/luciferase (pCRE-luc) (28), a cAMP-inducible luciferase-expressing plasmid. Stable cell lines expressing the wild-type or the different mutant receptors were transfected with pCRE-luc using Exgen 500 (Euromedex, Souffel Weyershiem, France). Five micrograms of pCRE-luc DNA were used for transfection of a $10-\mathrm{cm}$ dish of cells. At 24 hours after transfection, cells were split into one 48-well plate and incubated in $\mathrm{MEM} \alpha$ medium. At 48 hours after transfection, cells were washed and incubated in stimulation medium (MEM $\alpha$ medium containing $0.1 \mathrm{mg} / \mathrm{mL}$ BSA and 0.25 mM 3-isobutyl-1-methylxanthine) and stimulated with different concentrations of $\alpha-\mathrm{MSH}$ (Sigma Chemical Co., St. Louis, Missouri, USA) for 6 hours at $37^{\circ} \mathrm{C}$ in a $5 \% \mathrm{CO}_{2}$ incubator. The cells were also stimulated with $1 \mathrm{mM} 8 \mathrm{Br}$-cAMP to normalize for transfection efficiency. After stimulation, cells were lysed in $300 \mu \mathrm{L}$ of lysis buffer (25 mM glygly, $15 \mathrm{mM} \mathrm{MgSO}_{4}, 4 \mathrm{mM}$ EGTA, 1 mM DTT, 1\% Triton), frozen and thawed, and then assayed for luciferase activity using the Luciferase Reporter 1000 Assay System (Promega, Madison, Wisconsin, USA) in a luminometer as recommended by the manufacturer. Luciferase activity was normalized to protein concentration and displayed as a fold of $8 \mathrm{Br}-$
cAMP-stimulated level to normalize for efficiency of transfection of the pCRE-luc construct. Data represent means and standard deviation from five points; curves were fitted by nonlinear regression using the JMP3 software (SAS Institute Inc., Cary, North Carolina, USA).

Competition-binding experiments were performed on stable cell lines as follows: cells were plated at $5 \times 10^{5}$ cells per well in 48-well plates the day before the binding experiment was performed. Cells were washed with $200 \mu \mathrm{L}$ of binding medium $(1 \mathrm{mg} / \mathrm{mL}$ BSA in $\left.\mathrm{Ca}^{2+} / \mathrm{Mg}^{2+} \mathrm{PBS}\right)$ and incubated in $150-\mu \mathrm{L}$ binding medium containing 40,000-60,000 cpm of [125I] NDP-

MSH (NEN, Billerica, Massachusetts, USA) per well. Series concentration of unlabeled $\alpha-\mathrm{MSH}$ were used to compete with the labeled NDP- MSH. Controls for nonspecific binding contained $10 \mu \mathrm{M}$ unlabeled $\alpha-$ MSH. After 1 hour of incubation, the binding medium was aspirated, cells were washed with $400 \mu \mathrm{L}$ of binding medium, and $200 \mu \mathrm{L}$ of $0.1 \mathrm{~N} \mathrm{NaOH}$ was added. Membrane-bound counts per minute were determined in a Cobra gamma counter (Packard, Downers Grove, Illinois, USA). Total specific binding and $\mathrm{IC}_{50}$ values were determined by nonlinear regression analysis from triplicate data points using Prism software (GraphPad Software for Science, San Diego, California, USA).

Transient-expression assays. For the transient luciferaseexpression assays, HEK293 cells were cotransfected with pCRE-luc and either of two different constructs expressing wild-type or mutant MC4-R in a 6-well plate using Effectene. After 24 hours, cells from one well were split into 8 wells from a 48-well plate. At 48 hours after transfection, luciferase and binding assays were performed as described above for the stably transfected cells.

Genetic and phenotypic analysis of family 1417 . The Genebridge 4 radiation panel (Research Genetics, Huntsville, Alabama, USA) was screened with primer pairs MC4CF/MC4CR and MC4DF/MC4ER using the same conditions as for the PCR-SSCP. Out of 93 clones, 29 were positive, 61 were negative, and 3 were inconsistent. Data were submitted to World Wide Web to http://www-genome.wi.mit.edu/cgibin/contig/rhmapper.pl, at the Whitehead Institute (Cambridge, Massachusetts, USA) for computational and statistical analysis. MC4-R was mapped on the chromosome 18 framework map at 0cR 3000 from the STS WI-4461 (lod > 3). WI-4461 had been unambiguously assigned to YAC $938^{\mathrm{e}} 1$ (Whitehead Institute). Physical mapping of MC4-R to this YAC (Fondation Jean Dausset, Paris, France) was confirmed by PCR. Since the microsatellite marker D18S64 also mapped to the same YAC, we used this marker, as well as the two adjacent markers, D18S474 and D18S68, from the PE ABI panel (PE Biosystems, Foster City, California, USA) to genotype family 1417 at the MC4-R locus as described (29). For fluorescence in situ hybridization (FISH) analysis of the MC4-R locus in the proband, purified YAC $938^{\mathrm{e}} 1$ was labeled with biotin by nick translation (Boehringer Mannheim, Mannheim, Germany), following the manufacturer's instructions, and used as a probe. The FISH 
assay was carried out as described (30). Briefly, $100 \mathrm{ng}$ of biotin-labeled DNA probe was hybridized to chromosomes and signals detected with FITC-conjugated avidin. Forty metaphases were analyzed. Southern blot analysis of MC4-R was performed as described (31). Genomic DNA from the proband and from one control individual was digested with the restriction enzymes EcoRI, PstI, BstNI, and BanII. The probe encompassed $600 \mathrm{bp}$ of the $5^{\prime}$ untranslated region of the human MC4-R gene.

\section{Results}

Frequency of MC4-R mutations and association with human morbid obesity. Two hundred nine morbidly obese patients (BMI $>40 \mathrm{~kg} / \mathrm{m}^{2}$ ) were screened for mutations in the MC4-R gene by PCR-SSCP under conditions reported to be more than $90 \%$ sensitive for detecting single-base substitutions (32). Reamplification and direct sequencing determined the sequence of each detected variant. We found 11 different variants, encoding nine missense, one frameshift, and one silent mutation (Table 1). Each mutation was confirmed by sequencing the entire cloned MC4-R sequence of every mutation carrier. Mutation-specific PCR-RFLP assays were designed to assess the frequency of the missense and frameshift mutations in 254 nonobese controls (control 1 group). Two missense mutations described previously $(14,33)$, Val103Ile and Ile251Leu, were found at a similar frequency both in this control group and in the obese populations. No association of these polymorphisms with any obesity-related phenotype was observed in either group [data not shown]. The other mutations were not detected in the control 1 group.

To evaluate the significance of the high frequency of rare missense mutations in the obese cohort we searched for the presence of rare variants in a cohort of 112 nonobese nondiabetic controls (control 2 group). The entire MC4- $R$ coding sequence of these individuals was screened for mutation by PCR-SSCP. Whereas eight different rare mutations (seven missense and a new frameshift mutation) had been found in the 209 obese patients, no additional nonpolymorphic mutations were detected in the 112 controls. Table 1 summarizes the sequence and frequencies of the variants found in the three different cohorts. Taken together, our results indicate an association of rare mutations in $M C 4-R$ with morbid obesity $(P=0.03$, Fisher exact test).

Functional heterogeneity of buman obesity-associated MC4$R$ missense mutations. We investigated the pathogenic role of the missense mutations found in the morbidly obese cohort by comparing the activity of the different mutated MC4-Rs to that of the wild-type receptor. The wild-type and all mutated receptors were cloned, expressed in HEK 293 cells, and compared for their ability to bind and to be activated by their natural agonist $\alpha-M S H$. A cAMP-dependent luciferase assay was used to globally asses the activity of the Gs-coupled MC4-R in stably transfected cells expressing equivalent amounts of receptor.
In this assay, both the wild-type MC4-R and the MC4$\mathrm{R}$ containing the polymorphic amino acid change Ile251Leu exhibited a similar dose-response pattern curve (Figure 1a). A similar result, confirming data published previously (15), was found for the other polymorphic change, Val103Ile (not shown).

Mutations Thr150Ile, Arg165Trp, Ile170Val, and Ile301Thr all led to a reduction in receptor activation in our assay (Figure 1b). This result could be due to reduced expression of these mutated receptors at the cell membrane, reduced affinity for the ligand, or defective integral membrane function of the mutated receptor. To further explore the defects caused by these mutants we evaluated the affinity for ligand of cells expressing these mutated receptors in a binding assay. In the presence of subsaturating amounts $(200 \mathrm{pM})$ of $\left[{ }^{125} \mathrm{I}\right] \mathrm{NDP}-\alpha \mathrm{MSH}$, specific binding to the stably transfected (not shown) and transiently transfected cells expressing the mutants Arg165Trp, Ile170Val, or Ile301Thr were reduced by over $80 \%$, as compared with wild-type cells indicative of a strong reduction in cellsurface expression and/or ligand affinity of these mutated receptors (Figure 1e). Mutation Thr150Ile led to an intermediate reduction in receptor binding (Figure 1e), both in transiently and stably transfected cells. In a competition-binding assay this mutation caused an increase in the $\mathrm{IC}_{50}$ for $\alpha-\mathrm{MSH}$, as compared with the wild-type receptor (Figure 1f).

Mutation Leu250Gln displayed an unexpected pattern of activation. This receptor showed a major increase in basal activity (Figure 1c) and full activation by $\alpha$-MSH. This result was confirmed by comparing the activity of this mutant to the activity of the wildtype MC4-R under basal conditions in a transient assay (data not shown). This receptor has a 10-fold higher affinity for $\alpha-\mathrm{MSH}$, as determined in a competitionbinding assay (Figure 1f).

Mutations Arg18Cys and Thr11Ser, both changing amino acids in the extracellular $\mathrm{NH}_{2}$-terminal portion of MC4-R, did not have a major impact on the activity of the receptor in our cAMP-dependent luciferase assay (Figure 1d) nor on the affinity for $\alpha-\mathrm{MSH}$ in a competition-binding assay (Figure 1f).

Clinical characteristics of morbidly obese patients carrying $M C 4-R$ mutations. To search for specific phenotypes in the newly discovered MC4-R mutation carriers, we compared the clinical and biological characteristics of these patients with those of the other morbidly obese subjects screened for mutation as controls (Table 2). Mean BMI, maximal BMI reached during adult life, and minimal BMI reached after periods of caloric restriction were similar between both mutated and nonmutated groups $(P=0.6$ and $P=0.5$, respectively). In contrast, retrospective weight history indicates that the obese carriers of MC4-R mutations have an increased tendency to childhood obesity as compared with the other screened subjects (67 vs. $45.5 \%, P=$ 0.07 , respectively) and had a higher standard deviation of BMI at age 20 (Z-score at 20 years: $3.5 \pm 3.5$ vs. 
$1.4 \pm 2.3, P=0.04)$. The food intake assessed by dietary history was similar between groups as were the restraint, disinhibition, and hunger factors, suggesting no quantitative or qualitative modification of food intake behavior due to MC4-R mutation. The proportions of type 2 diabetic ( 27.0 vs. $25.0 \%$ ) or glucose-intolerant subjects (16 vs. $12.5 \%$ ) were not statistically different between both nonmutated and mutated obese groups $(P=0.6)$. As indicated by homeostatic model assessment of fasting glucose and insulin (23), nondiabetic obese subjects were markedly insulin resistant when compared with control group 1, but no difference was seen between MC4-R mutation bearers and nonbearers (data not shown). Fasting insulin and triglyceridemia were raised in both obese groups compared with the control $(P<$ 0.05 ), but were similar between obese groups. In addition, no difference was seen for the sex-adjusted mean leptin in both groups $(P=0.7)$. Hormone measurements were performed in three individuals with MC4$\mathrm{R}$ mutations. The corticotropic axis and thyrotropic axis of the proband carrying the 47-48insG mutation were normal (0800 hours: $163 \mathrm{ng} / \mathrm{mL}$ [110 to 250 ], 1600 hours: $75 \mathrm{ng} / \mathrm{mL}$ [20 to 90], after dectancyl < 10 $\mathrm{ng} / \mathrm{mL} ; \mathrm{T} 4=7.6 \mathrm{pg} / \mathrm{mL}, \mathrm{TSH}=3.6 \mathrm{mUI} / \mathrm{mL}$ ). Similarly, the probands carrying the Leu250Gln and the Arg165Trp mutations had normal cortisol and thyroid hormones levels.

Variable penetrance and expressivity of buman MC4-R mutations. We evaluated the penetrance and expressivity of the rare MC4-R mutations in all available family members of the probands. A total of 29 family members were screened for mutation using the mutationspecific PCR-RFLP assays designed for the association studies. Carriers of MC4-R mutations were found in nine subjects from four families (Figure 2). In the families 1143 (Figure 2a), 1261 (Figure 2b), and 1283 (Figure 2c), segregating mutations Arg165Trp, Ile170Val, and Ile301Thr, respectively, all the mutation carriers had a history of obesity, but the age of onset and the severity of obesity varied between and within these families. In sharp contrast, the three MC4-R-mutation carriers in family 1417 had no history of obesity (Figure 2e). The MC4-R mutation segregating in this family is the new MC4-R 47-48insG frameshift mutation. This mutation results in the expression of an aberrant peptide containing the first 16 amino acids of MC4-R and 12 additional amino acids. True haploinsufficiency therefore should be expected in the carriers of this mutation. Careful clinical assessment of the nonobese heterozygous MC4-R 47-48insG carriers of family 1417 did not show any findings that explain the incomplete penetrance of obesity in these individuals (Figure 2e).

The hypothesis of a recessive transmission of obesity linked to $M C 4-R$ was evaluated in family 1417 . Genotyping at the $M C 4-R$ locus indicated that the proband shared the same haplotypes as her younger lean brother. FISH analysis did not show any chromosomal rearrangement of the MC4- $R$ locus in the proband.
Southern blots using multiple restriction enzymes did not show evidence for a deletion at the MC4-R locus. Sequence of the entire MC4- $R$ gene, including the $5^{\prime}$ and $3^{\prime}$ untranslated regions, did not show a second MC4-R mutation in the proband.

Variable penetrance of obesity associated with MC4$\mathrm{R}$ mutations in family 1417, as well as variable expressivity of obesity associated with MC4-R mutations in other families, could be due to genetic variations in the MC4-R ligand genes. One hundred morbidly obese patients from the same cohort as that screened for MC4-R mutations were screened for mutation by PCRSSCP in the three exons encoding the AGRP gene and in the $\alpha$-MSH-encoding portion of the POMC gene. No variants were found in the $\alpha$-MSH-encoding portion of the POMC gene in all the tested subjects. Two silent mutations (G423A and C690T) were found in the AGPR-encoding sequence of two different patients.

\section{Discussion}

Polygenic metabolic diseases, such as obesity, have been suggested to result from the additive and interactive effects of a limited number of common genetic variants (5). Alternatively, the predisposition to excessive weight gain in a given environment could depend upon multiple rare mutations (with different penetrance) in a large set of genes (5). This study provides evidence that $M C 4-R$ is the first of such genes. MC4-R shows a higher mutation frequency in common morbid obesity than in normal-weight control individuals. This significant trend is observed across studies. Including ours, five groups have independently screened the MC4- $R$ for mutations in a total of 1,136 patients $(12-15,33) ; 771$ were extremely obese (weight $>99$ th percentile in children or BMI $>40 \mathrm{~kg} / \mathrm{m}^{2}$ in adults), and 315 had a BMI below 30 (50 had unknown or intermediate BMI). Three missense variants, Val103Ile, Thr112Met, and Ile251Leu, and one silent mutation were found both in extremely obese and lean individuals. In contrast, whereas a total of 15 mutated $M C 4-R$ alleles have been

\section{Table 1}

MC4-R mutation screening in morbidly obese patients and nonobese controls

\begin{tabular}{lcccc}
\hline Base change & $\begin{array}{c}\text { Effect on } \\
\text { amino acid } \\
\text { sequence }\end{array}$ & $\begin{array}{c}\text { Morbidly obese Control 1 } \\
(n=209)\end{array}$ & $\begin{array}{c}\text { Control 2 } \\
(n=254)\end{array}$ & $(n=112$ \\
A-307-G & Val103 lle & 8 & 8 & 3 \\
A-751-C & Ile251Leu & 3 & 3 & 0 \\
C-593-T & Silent & 1 & ND & 1 \\
47-48insG & 16+12 amino-acids & 1 & 0 & 0 \\
A-31-G & Thr11Ser & 1 & 0 & 0 \\
C-52-T & Arg18Cys & 1 & 0 & 0 \\
C-449-T & Thr150lle & 1 & 0 & 0 \\
A-508-G & Ile170Val & 1 & 0 & 0 \\
C-493-T & Arg165Trp & 1 & 0 & 0 \\
T-749-A & Leu250Gln & 1 & 0 & 0 \\
T-902-C & Ile301Thr & 1 & 0 & 0
\end{tabular}

The morbidly obese and the control 2 populations were screened by PCR-SSCP. The control 1 population was screened by PCR-RFLP for every functionally relevant mutation detected in the morbidly obese population. ND, not determined. 


\section{Figure 1}

Function of obesity-associated MC4-R mutants. (a-d) Activity of the receptors is assayed by analyzing their ability to activate expression of a cAMPinduced luciferase fusion gene. Cells stably expressing each receptor and transiently expressing the fusion construct were stimulated for 6 hours with medium alone, increasing amounts of $\alpha-\mathrm{MSH}$ or $8 \mathrm{Br}$-cAMP, after which luciferase activity was measured. Data points represent means of five determinations divided by maximal levels of luciferase activity achieved by $1 \mathrm{mM}$ 8Br-cAMP (average of five determinations). Error bars indicate SD. The wildtype receptor activation curve is shown on all graphs for comparison with: (a) the polymorphic lle251 Leu mutation; (b) mutations Thr150lle, Arg165Trp, Ile170Val, and lle301Thr; (c) mutation Leu250Gln; (d) the $\mathrm{NH}_{2}$-terminal Thr11Ser and Arg18Cys mutations. The $\mathrm{EC}_{50} \pm \mathrm{SD}$ is indicated in parentheses for each variant receptor. (e) Binding of [125I] NDP- $\alpha-M S H$ to 293 cells transiently transfected with the wild-type and mutant MC4-Rs. Cells were incubated with a subsaturating amount of [125I]labeled NDP- $\alpha-M S H$ (200 pM) under conditions described in Methods. The specific binding was derived from the total binding minus nonspecific binding. Data points represent the mean of three independent experiments performed in triplicate and normalized to wild-type binding for each experiment. Error bars indicate SD. (f) Competitionbinding assay. Stably transfected cells were incubated with [ ${ }^{125}$ I] NDP- $\alpha-M S H$ in the presence of increasing concentrations of $\alpha-\mathrm{MSH}$. All curves are representative of three different experiments and each point is the mean of triplicate values. Error bars indicate SD. The ordinate is expressed as a percentage of total specific binding $\left(B_{\max }\right)$. Curves are fitted using nonlinear regression analysis and a onesite competition model (GraphPad Prism). The IC $\mathrm{C}_{50}$ $\pm \mathrm{SD}$ is indicated in parentheses for each receptor.

\section{a}

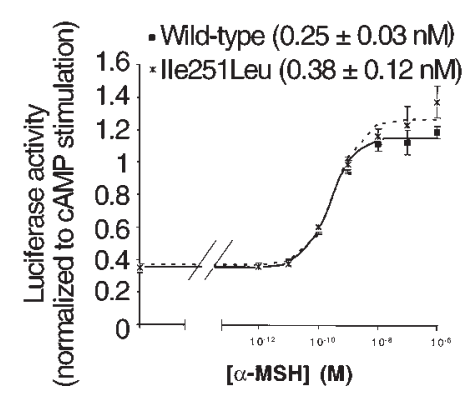

c
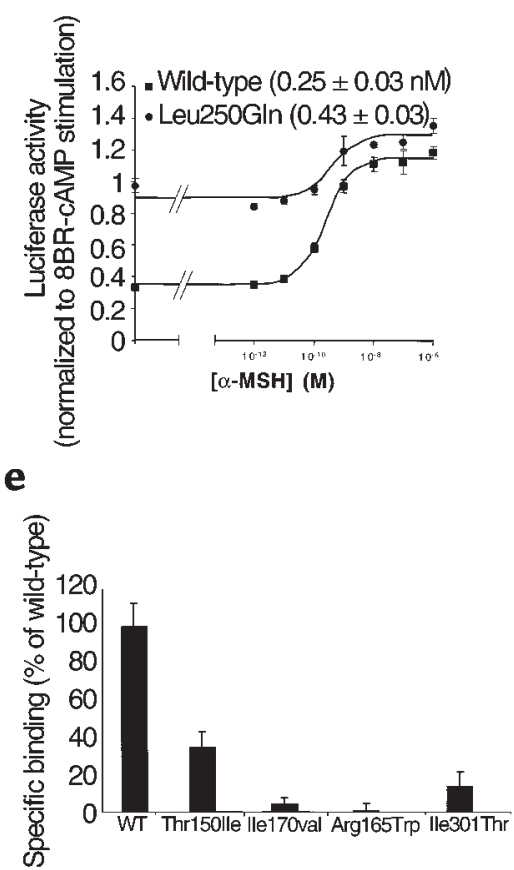

b

- Wild-type $(0.25 \pm 0.03 \mathrm{nM})$

- lle301Thr $(1.10 \pm 0.12 \mathrm{nM})$

× Thr150lle $(5.97 \pm 0.54 \mathrm{nM})$

- lle170Val $(7.26 \pm 0.95 \mathrm{nM})$

- $\operatorname{Arg} 165 \operatorname{Trp}(4.97 \pm 1 \mathrm{nM})$

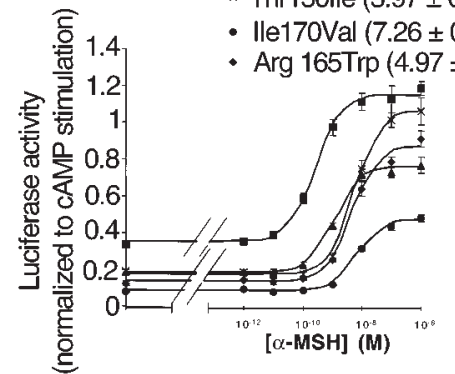

- Wild-type $(0.25 \pm 0.03 \mathrm{nM})$

d $\quad$ * lle301Thr $(1.10 \pm 0.12 \mathrm{nM})$
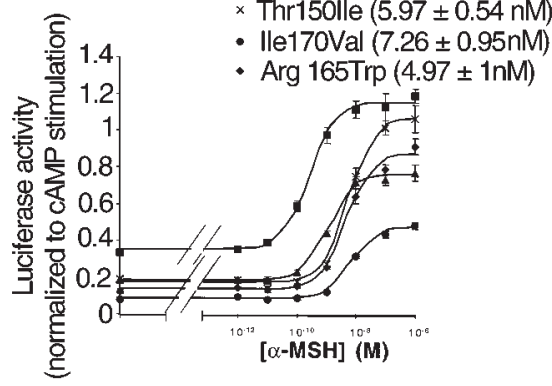

f

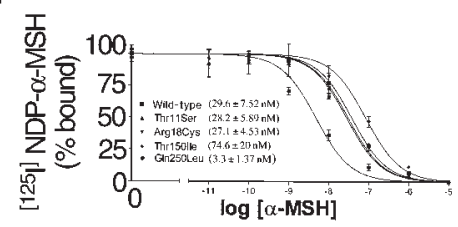

found only in 18 extremely obese probands $(2.3 \%$ of $771)$, no additional mutation has been found only in the 315 lean individuals $(P<0.005$, Fisher exact test). The high frequency (4\%) of rare MC4-R mutations found in our French morbidly obese population does not significantly differ from that of previously screened morbid obese populations, but confirms that MC4-R mutations are implicated in a large absolute number of cases of morbid obesity. Indeed, since $0.5 \%$ of the French population has a BMI above $40 \mathrm{~kg} / \mathrm{m}^{2}$ (34), our results suggest that the prevalence of morbid obesity due to MC4-R mutation in the French population could be close to 1 in 5,000.

Previously, rare monogenic forms of human obesity have been identified through associated endocrine abnormalities (7-11). In contrast, by extensively phenotyping and comparing MC4-R mutation carriers with non-mutation carriers, we did not detect any specific clinical or biological symptoms characterizing the MC4-R mutation bearers, except for a trend toward earlier onset and more severe obesity at age 20 . In the sub- group of patients presenting with a sex-specific standard deviation of BMI above 1.5 at age 20 , the frequency of rare mutations in MC4-R was $6 \%$. Whereas this finding is consistent with the frequency of MC4-R mutations found in obese children selected with a lower cut-off point for obesity (14), additional studies in larger cohorts will be necessary to confirm the increased sensitivity brought by this diagnostic criteria.

Confirmation of the pathogenic role of missense mutations involves functional characterization of the mutant receptor. By systematically assaying the function of the different mutant receptors in a cell base system, we found that all but two of the obesity-associated missense mutations impaired the function of the receptor in our assay whereas polymorphic variants of the receptor did not. This result strengthens the epidemiological argument for a role of these MC4-R mutations in the obese phenotype of the probands and again underscores results obtained in previous studies. Indeed, the one nonsense (Tyr-35-Stop) (14) and the three frameshift mutations (732-733insGATT, 631-634delCTCT, 47- 
48insG) (refs. 12, 13, and this study) described in the MC4-R can be considered loss-of-function mutations. A previously studied mutation (Ile137Thr) has also been shown to impair the function of MC4-R (15). Together, 11 out of the 15 mutated MC4-R alleles found in 14 out of 18 extremely obese individuals have a significant impact on receptor function.

a

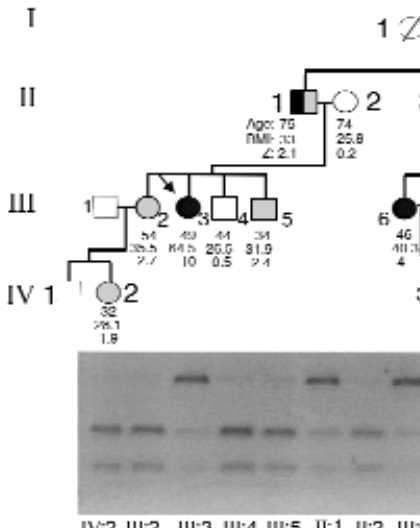

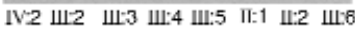
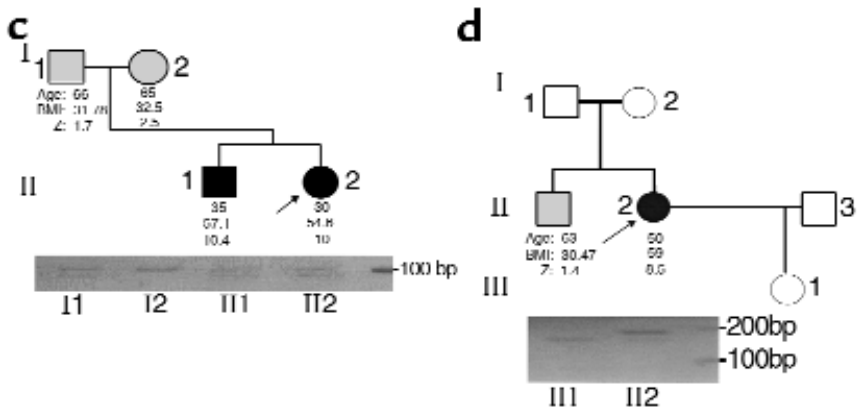

The two mutations (Thr11Ser and Arg18Cys) found in morbidly obese individuals that did not significantly modify the function of MC4-R are located in the $\mathrm{NH}_{2}$-terminal region of the protein. This $\mathrm{NH}_{2}$-terminal region of MC4-R can be deleted without affecting ligand binding (35). It is possible that this region is necessary for another property of the receptor that might

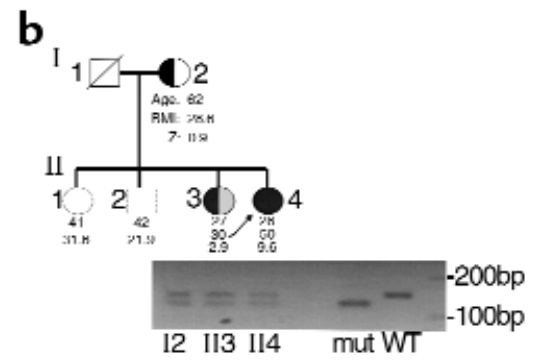

$-300 \mathrm{bp}$

$-200 \mathrm{bp}$

$-100 \mathrm{bp}$

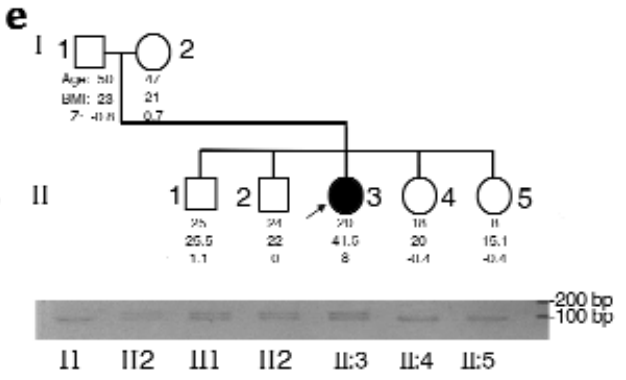

\section{Figure 2}

Pedigrees, mutation screening, and phenotypes in the available family members of MC4-R mutation carriers. For each mutation, the upper part of the figure represents the pedigree structure of the family of the carrier. Age (years), BMI $\left(\mathrm{kg} / \mathrm{m}^{2}\right)$, and Z-score (standard deviation of $\mathrm{BMI})$ are indicated under each individual. Arrows indicate the probands. Hatched symbols denote obesity $(30 \leq \mathrm{BMI}<40 \mathrm{~kg} / \mathrm{m} 2)$. Filled symbols denote early-onset morbid obesity (BMI $\geq 40$ ). Half-filled symbols denote a history of childhood obesity (obesity before age 20 ). The lower portion of each part of the figure represents the result of PCR-RFLP genotyping of each available family member. Genotyping was performed using a specific assay for each mutation, as described in Methods. C+: positive control (amplified from the cloned mutation and digested); C-: negative control (amplified from the wild-type receptor and digested). (a) In family 1143 the Arg165Trp mutation was transmitted to the proband III:3 by her obese father II:1. He developed obesity during infancy (BMI $=31 \mathrm{~kg} / \mathrm{m}^{2}$ at 16 years, Z-score $=4.6 \mathrm{SDs}$ of $\mathrm{BMI}$ ). During adulthood, he reached the diagnostic cut-off point for morbid obesity, since his maximal lifetime BMI was $41 \mathrm{~kg} / \mathrm{m}^{2}$ at age 30. His lifetime weight variation was $90-125 \mathrm{~kg}$. The proband's cousin III:6, bearing the mutation, also had a morbid form of obesity of early onset ( $80 \mathrm{~kg}$ at age 10 years and $100 \mathrm{~kg}$ at age 16 years). Her mother, II:3, closely resembled proband III:3, but no precise phenotypic data were available. (b) In family 1261 the mutation Ile170Val was transmitted by the obese mother I:2, who developed obesity during puberty (at 14 years of age, BMI $28 \mathrm{~kg} / \mathrm{m}^{2}$, Z-score $=3$ ). Her adult lifetime weight variation was $50-90 \mathrm{~kg}$. She also transmitted the mutation to her daughter II:3, who became overweight at puberty (at 14 years old, BMI $=28 \mathrm{~kg} / \mathrm{m}^{2}$, Z-score $=3.5$ ), and her BMI was $30.4 \mathrm{~kg} / \mathrm{m}^{2}(Z$-score $=$ 3.8 ) at age 20. Her maximal weight was $82 \mathrm{~kg}$ after the age of 20. (c) In family 1283 all the carriers of mutation lle301Thr were obese. The proband's brother is a morbidly obese man (BMI 57.1, percent body fat 54\%), with severe obesity developed early in infancy. His birth weight was normal $(2.8 \mathrm{~kg})$. He had a BMI of $22 \mathrm{~kg} / \mathrm{m}^{2}(Z$-score $=4.3)$ at 9 years old, and he was morbidly obese $\left(\mathrm{BMl} 47 \mathrm{~kg} / \mathrm{m}^{2}, \mathrm{Z}\right.$-score $\left.=13\right)$ at 14 years. His adult lifetime weight variation was between 117 and $185 \mathrm{~kg}$. His obesity is complicated by glucose intolerance and hypertriglyceridemia. The mutation was transmitted to this sibling pair by the father l:1, who had a less-severe form of obesity of later onset (after the age of 20 years). (d) In family 1413, no clinical nor genotype data were available for the parents of the proband II:2 carrying the constitutively active Leu250GIn mutation. Her brother II:1 is not carrying the mutation. (e) In family 1417 the 47-48insG frameshift mutation was transmitted to the proband II:3 by her nonobese mother I:2 (BMI $\left.=21 \mathrm{~kg} / \mathrm{m}^{2}\right)$. She never had weight problems either during infancy or during puberty, and her maximal reached BMI was $22 \mathrm{~kg} / \mathrm{m}^{2}$. She moderately controls her body weight, with declared diet restriction below one per year, and is not a sportswoman. She also transmitted the mutation to her two older sons, II: 1 and II:2. Both are nonobese young males, 25 and 24 years old, with a BMI of 25.5 and $22 \mathrm{~kg} / \mathrm{m}^{2}$, respectively. 


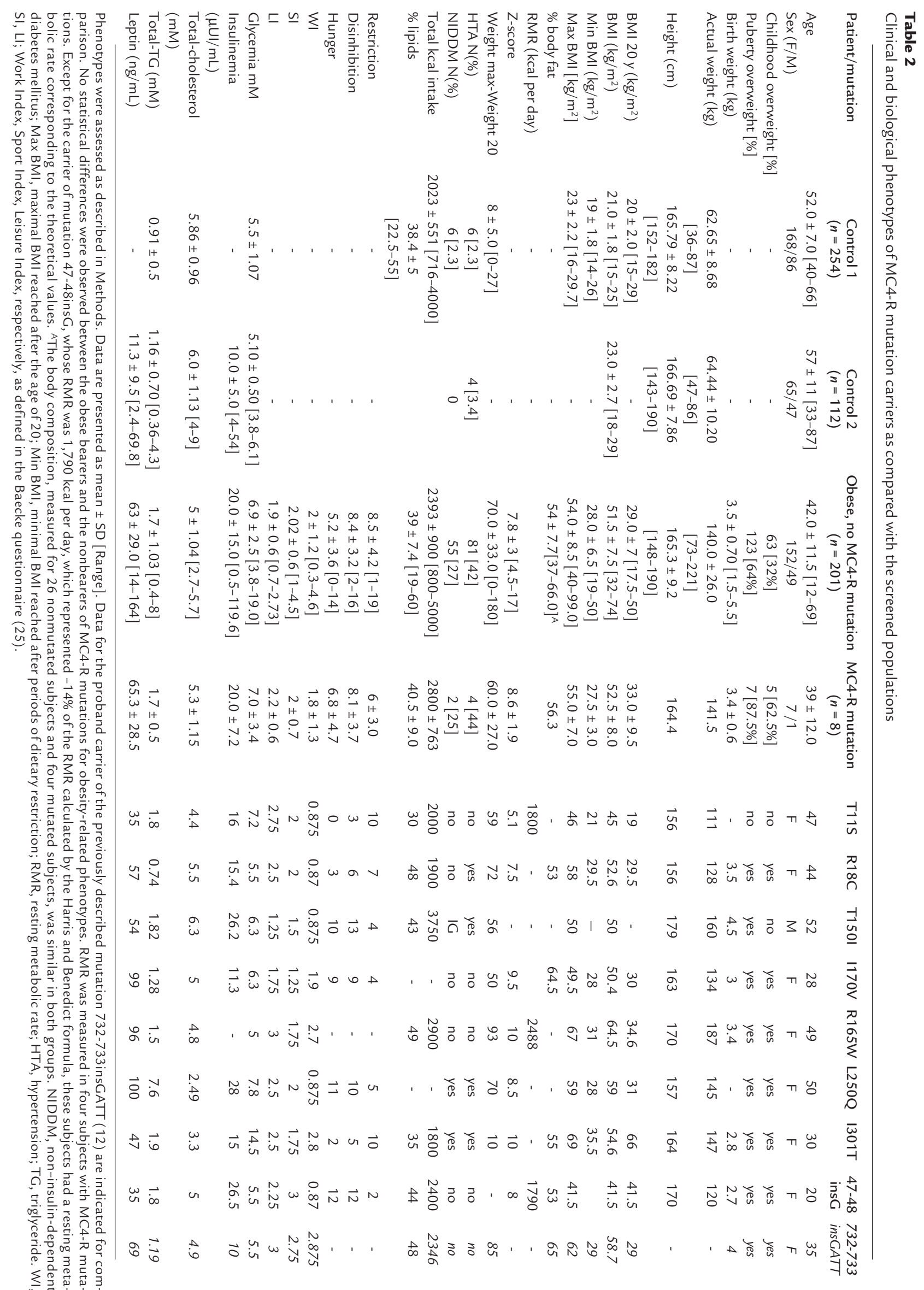


not be evaluated through our assays. Additional studies will be required to dismiss any functional effects for mutations Thr11Ser and Arg18Cys.

The Leu250Gln mutation described in this study is the first obesity-associated mutation leading to constitutive activation of MC4-R. Association of this mutation with obesity is surprising since activation of MC4$\mathrm{R}$ should result in an increased satiety signal. Indeed, the proposed use of long-acting agonist of the MC4-R in the treatment of obesity should be reconsidered in view of this result. However, since constitutive activation in a cell-based assay might not accurately represent the in vivo behavior of this mutated receptor, additional studies are clearly required to precisely understand the role of the Leu250Gln MC4-R mutation in the obese phenotype of our proband.

Reduced penetrance and variable expressivity have been seen in most diseases linked to mutations in GPCRs (36). In this study we demonstrate, we believe for the first time, that heterozygous-null MC4-R mutations in humans do not always lead to obesity. Heterozygous $M C 4-R^{+/-}$mice also display a broad variability in phenotype with an adult weight ranging from that of wildtype to that of homozygous $M C 4-R^{-/-}$mice (22). Since the trait under consideration is a quantitative and environmentally dependent trait, a better estimation of the penetrance of obesity in MC4-R mutation carriers will require the review of large well-phenotyped pedigrees across multiple additional studies.

The reduced penetrance and variable expressivity of obesity associated with MC4-R mutations could be influenced by variants in modifier genes that are both intrinsic and extrinsic to the melanocortin pathway. We found no evidence for functionally significant variation in $\alpha-\mathrm{MSH}$ or AGRP, which are both intrinsic to the pathway, but these negative data should be interpreted with caution.

The reduced penetrance and variable expressivity of obesity observed between families in this study could also be linked to the differential functional effects of the receptor mutations. Since many of the previous mutations found in MC4- $R$ were expected to result in a truncated protein, it had been hypothesized that the obesity in the MC4-R mutation carriers resulted from haploinsufficiency $(13,14)$. In contrast, a recent study on a small population of patients with deletions of chromosome 18q, some of them hemizygous at the $M C 4-R$ locus, supports the hypothesis that the involvement of MC4-R in obesity may reflect a dominant-negative effect (37). In our study, both the reduced penetrance of obesity in heterozygous carriers of the MC4-R 47-48insG frameshift mutation and the constitutive activation observed for one of the missense mutants suggest that obesity associated with MC-4R mutations should not always be solely attributed to haploinsufficiency at this locus.

In summary, our results confirm that mutations in MC4-R are the most frequent genetic cause of common obesity described to date. The heterogeneity of obesity linked to MC4-R mutations clearly implies that functional assessment of MC4-R mutations will eventually routinely be required to evaluate the risk of obesity in MC4-R mutation carriers. In a broader perspective, this study suggests that the comparison of the occurrence of rare functionally relevant mutations between patient and control populations might represent the best method for implicating candidate genes in the pathogenesis of a multifactorial polygenic trait such as common obesity.

\section{Acknowledgments}

This work was supported by the Direction de la Recherche Clinique/Assistance Publique-Hopitaux de Paris, Programme Hospitalier de Recherche Clinique (AOM 96088), and a grant from Institut de Recherche Internationale Servier. Part of this work was supported by a Howard Hughes Medical Institute Research Resource Program grant (76296-549901) to University of California San Francisco School of Medicine. C. Vaisse was supported by l'Institut National de la Santé et de la Recherche Medicale. The Danone Institute is gratefully acknowledged for giving access to the Danone/Suvimax DNA bank. We thank Valerie Delannoy, Cytogenetics Laboratory of the Institute of Biology of Lille, Genevieve Bonhomme, and Annie Legal for their excellent technical help. We thank G. Barsh for critical reading and insightful comments on the manuscript.

1. Allison, D.B., Fontaine, K.R., Manson, J.E., Stevens, J., and VanItallie, T.B. 1999. Annual deaths attributable to obesity in the United States. JAMA. 282:1530-1538.

2. Must, A., et al. 1999. The disease burden associated with overweight and obesity. JAMA. 282:1523-1529.

3. 1998. Obesity: preventing and managing the global epidemic. World Health Organization. Geneva, Switzerland. Report no. WHO/NUT/NCD/98-1.276 pp.

4. Mokdad, A.H., et al. 1999. The spread of the obesity epidemic in the United States, 1991-1998. JAMA. 282:1519-1522.

5. Comuzzie, A.G., and Allison, D.B. 1998. The search for human obesity genes. Science. 280:1374-1377.

6. Hill, J.O., and Peters, J.C. 1998. Environmental contributions to the obesity epidemic. Science. 280:1371-1374.

7. Montague, C.T., et al. 1997. Congenital leptin deficiency is associated with severe early-onset obesity in humans. Nature. 387:903-908.

8. Strobel, A., Issad, T., Camoin, L., Ozata, M., and Strosberg, A.D. 1998. A leptin missense mutation associated with hypogonadism and morbid obesity. Nat. Genet. 18:213-215.

9. Clement, K., et al. 1998. A mutation in the human leptin receptor gene causes obesity and pituitary dysfunction. Nature. 392:398-401.

10. Krude, H., et al. 1998. Severe early-onset obesity, adrenal insufficiency and red hair pigmentation caused by POMC mutations in humans. Nat. Genet. 19:155-157.

11. Jackson, R.S., et al. 1997. Obesity and impaired prohormone processing associated with mutations in the human prohormone convertase 1 gene. Nat. Genet. 16:303-306.

12. Vaisse, C., Clement, K., Guy-Grand, B., and Froguel, P. 1998. A frameshift mutation in human MC4R is associated with a dominant form of obesity. Nat. Genet. 20:113-114.

13. Yeo, G.S., et al. 1998. A frameshift mutation in MC4R associated with dominantly inherited human obesity. Nat. Genet. 20:111-112.

14. Hinney, A., et al. 1999. Several mutations in the melanocortin-4 receptor gene including a nonsense and a frameshift mutation associated with dominantly inherited obesity in humans. J. Clin. Endocrinol. Metab. 84:1483-1486.

15. Gu, W., et al. 1999. Identification and functional analysis of novel human melanocortin-4 receptor variants. Diabetes. 48:635-639.

16. Gantz, I., et al. 1993. Molecular cloning, expression, and gene localization of a fourth melanocortin receptor. J. Biol. Chem. 268:15174-15179.

17. Sundaramurthy, D., Campbell, D.A., Leek, J.P., Markham, A.F., and Pieri, L.F. 1998. Assignment of the melanocortin 4 receptor (MC4R) gene to 
human chromosome band $18 \mathrm{q} 22$ by in situ hybridisation and radiation hybrid mapping. Cytogenet. Cell Genet. 82:97-98.

18. Fan, W., Boston, B.A., Kesterson, R.A., Hruby, V.J., and Cone, R.D. 1997. Role of melanocortinergic neurons in feeding and the agouti obesity syndrome. Nature. 385:165-168.

19. Rouille, Y., et al. 1995. Proteolytic processing mechanisms in the biosynthesis of neuroendocrine peptides: the subtilisin-like proprotein convertases. Front. Neuroendocrinol. 16:322-361.

20. Ollmann, M.M., et al. 1997. Antagonism of central melanocortin receptors in vitro and in vivo by agouti-related protein. Science. 278:135-138.

21. Graham, M., Shutter, J.R., Sarmiento, U., Sarosi, I., and Stark, K.L. 1997. Overexpression of Agrt leads to obesity in transgenic mice. Nat. Genet. 17:273-274.

22. Huszar, D., et al. 1997. Targeted disruption of the melanocortin-4 receptor results in obesity in mice. Cell. 88:131-141.

23. Clément, K., et al. 1997. Association of poorly controlled diabetes with low serum leptin in morbid obesity. Int. J. Obes. 21:556-561.

24. Stunkard, A.J., and Messick, S. 1985. The three-factor eating questionnaire to measure dietary restraint, disinhibition and hunger. J. Psychosom. Res. 29:71-83.

25. Baecke, J.A., Burema, J., and Frijters, J.E. 1982. A short questionnaire for the measurement of habitual physical activity in epidemiological studies. Am. J. Clin. Nutr. 36:936-942.

26. Hercberg, S., et al. 1998. A primary prevention trial using nutritional doses of antioxidant vitamins and minerals in cardiovascular diseases and cancers in a general population: the SU-VI-MAX study-design, methods, and participants characteristics. Control. Clin. Trials. 19:336-351.

27. Lu, D., Vage, D.I., and Cone, R.D. 1998. A ligand-mimetic model for con- stitutive activation of the melanocortin-1 receptor. Mol. Endocrinol. 12:592-604

28. Spengler, D., et al. 1993. Differential signal transduction by five splice variants of the PACAP receptor. Nature. 365:170-175.

29. Hager, J., et al. 1998. A genome-wide scan for human obesity genes reveals a major susceptibility locus on chromosome 10. Nat. Genet. 20:304-308.

30. Delobel, B., et al. 1998. Identification and molecular characterization of a small $11 \mathrm{q} 23.3$ de novo duplication in a patient with Rett syndrome manifestations. Am. J. Med. Genet. 80:273-280.

31. Vaisse, C., Atger, M., Potier, B., and Milgrom, E. 1990. Human placental protein 14 gene: sequence and characterization of a short duplication. DNA Cell Biol. 9:401-413.

32. Grompe, M. 1993. The rapid detection of unknown mutations in nucleic acids. Nat. Genet. 5:111-117.

33. Gotoda, T., Scott, J., and Aitman, T.J. 1997. Molecular screening of the human melanocortin- 4 receptor gene: identification of a missense variant showing no association with obesity, plasma glucose, or insulin. Diabetologia. 40:976-979.

34. Rolland-Cachera, M.F., et al. 1991. Body Mass Index variations: centiles from birth to 87 years. Eur. J. Clin. Nutr. 45:13-21.

35. Schioth, H.B., Petersson, S., Muceniece, R., Szardenings, M., and Wikberg, J.E. 1997. Deletions of the N-terminal regions of the human melanocortin receptors. FEBS Lett. 410:223-228.

36. Spiegel, A.M. 1998. G proteins, receptors, and disease. Humana Press. Totowa, New Jersey, USA. 324 pp.

37. Cody, J.D., et al. 1999. Haplosufficiency of the melanocortin-4 receptor gene in individuals with deletions of 18q. Hum. Genet. 105:424-427. 